\title{
The Wisdom of Networked Evolving Agents
}

\author{
Akira Namatame \\ Department of Computer Science, National Defense Academy, Yokosuka, \\ Kanagawa, 239-8686, Japan \\ nama@nda.ac.jp, http://www.nda.ac.jp/ nama
}

Summary: The fact that selfish behavior may not achieve full efficiency at the collective level has been well also known in the literature. Therefore we need to cope with the socio-economic system by attempting to stack the deck in such a way that individuals with selfish incentives have to do what is the desirable thing. Of particular interests is the question how social interactions can be restructured so that agents are free to choose their own actions while avoiding outcomes that none would have chosen. In this paper, we study the collective construction process of social norms by networking evolving agents. Social norms are here treated as emerged behavioral rules that constitute constraints on social interactions so that they can achieve efficient and equitable outcomes.

\section{Introduction}

It is common in many markets that the buying decision of one consumer influences the decisions of others. The general effects applying to all the consumer decisions, markets also have strong positive or negative network effects. Popular examples of positive network effects are the willingness to adopt a product innovation correlates positively with the number of existing adopters. Positive network effects in markets mainly originate from two different areas, the need for compatibility to exchange information or data and the need for complementary products and services.

On the other hand, in many cases, the existence of network effects results in so called madness of crowd [1]. Economic implications resulting from the bandwagon and herding behavior are broadly discussed in literature. Network externalities also lead to Pareto-inferior outcomes due to coordination failure. The fact that selfish behavior may not achieve full efficiency at the collective level has been well also known in the literature. Recent research efforts have focused on quantifying the loss of system performance due to selfish and uncoordinated behavior. The degree of efficiency loss is also known as the price of anarchy [17]. The reason why uncoordinated activities of agents pursuing their 
Akira Namatame

own interests often produce outcomes that all would seek to avoid is that each agent's behavior affect the others and these effects are often not included in whatever optimizing process made by other agents. These unaccounted effects on others are called externalities

Socio-economic systems consist of individuals and the socio-economic system in which they interact. On the other side, a collective of individuals creates the socio-economic system of which they are parts. Therefore, the essence of the socio-economic system is that it is the individuals who are making their decisions. We need to cope with the socio-economic system by attempting to stack the deck in such a way that individuals have selfish incentives to do what is the desirable thing. Explicit or implicit coordination is necessary to achieve individuals' goals more efficiently. However, many aggregate social outcomes have emergent properties that cannot be trivially derived from the properties of their members.

In his book, titled The Wisdom Of Crowds, Surowiecki explores an idea that has profound implications: a large collection of people are smarter than an elite few, no matter how they are brilliant and better at solving problems, fostering innovation, coming to wise decisions, even predicting the future [18]. He explains the wisdom of crowds emerges only under the right conditions: (1) diversity, (2) independence, (3) decentralization, and (4) aggregation. His counterintuitive notion, rather than the madness of crowd such as herding, cascade as traditionally understood [1], suggests new insights for the issue on how complex social and economic activities should be organized.

This observation derives requirements for a more general model of network effects. Therefore a new area of research is emerged aiming at explaining the phenomena of strong positive or negative network effects in markets and their implications on market coordination and efficiency. A crucial ingredient in social interaction models is the network structure in which individuals interact. However, the assumptions and simplifications implicitly used for modeling social interaction processes fail to explain the individual cognitive decisionmaking process.

Many spheres of social interactions are governed by social norms such as reciprocity and equity. Social norms are self-enforcing patterns of social behavior. It is in everyone's interests to conform given the expectation that others are going to conform. It is a rule of strategy choice that assigns a rule to each agent that is an optimal in the sense no one has an incentive to deviate from it. Although social norms can potentially serve useful constructs to understand human behavior, there is little theory on evolutionary construction of social norm. Young worked on the evolutionary process that brings about norms [22]. He works on the model and discovers that, once people got the norm they are no longer trying to make decisions the way they make them before there was a norm. If the norm is a man always opens the door for a woman, once you have the 
norm pretty soon all children will grow up knowing the norm. Once people have norm they can internalize the norm, they can remember the norm, and they can teach the norm. If we want to see whether game theory can be of any help in thinking about which norms come about, how they come about, how durable they may be when they come about, then game theory can help.

We study emergence of social norms as the wisdom of a collective of agents. The social space consists of networks of self-interested agents, continuous evaluations of their performance as well as their sets of behavioral rules. Behavioral rules are here treated as the constraints on individual action and they specify the action choice based on the specific outcomes. The learning of new behavioral rule, and the strife of each agent to act in keeping with the coupling with the neighbors constitute the emergence of social norms. Social norms are here treated as the shared behavioral rules that constitute common constraints on all individuals in a society. For agents in a society to achieve social intelligence, it is a continuous process that requires social behavior based on social rationality [14]. To in turn achieve social rationality requires for individually rational behavioral rule to be constrained also by some obligations. We study collective construction of social norms by focusing on the relation between micro and macro levels of constraints on the evolution of socially intelligent behavior.

\section{Game Theoretic Models of Social Interaction}

The interaction structure specifies who affects whom, and this network structure may vary from one individual to another. Social interdependence can be understood as a dependence of outcomes of one individual on another individual behavior. Such a relationship between payoffs for choices of different individuals is usually described with the formalism of the game theory.

\subsection{Coordination Game: Nash Demand Game}

We begin by modeling a bargaining process between two agents. Consider two agents, A and B, each of whom demands some portion of a "pie", which we take as a metaphor for a piece of available resources which is divisible. A way of modeling this bargaining situation is the Nash demand game: each agent gets his demand if the sum of the two demands is not more than 10; otherwise each gets nothing. We simply assume that each agent can make just three possible demands: low (3), medium (5), and high (7). The payoffs (in share) from all combinations of demands are shown in Table 1 . This Nash demand game yields 
Akira Namatame

a coordination game in which there are three pure Nash equilibria: $\left(S_{1}, S_{3}\right),\left(S_{2}\right.$, $\left.\mathrm{S}_{2}\right)$, and $\left(\mathrm{S}_{3}, \mathrm{~S}_{1}\right)$.

Axtell and his colleagues explored which equilibrium emerges at the aggregate level from the repeated pair-wise interactions of self-interested agents [2]. They consider a population of $N$ agents and in each match, one pair of agents is drawn at random from the, and they play the game in Table 1. Therefore there is no network effect. Each agent makes a demand that maximizes her expected payoff (best-response) about the opponent's behavior, which defines the current state of the population. However, with some small probability, each agent chooses one of the three demands at random. They showed that the equity norm, corresponding to the equilibrium $\left(\mathrm{S}_{2}, \mathrm{~S}_{2}\right)$, and each agent demand medium has a large basin of attraction. In the terminology of evolutionary game theory, the equity norm is stochastically stable. Occasional random choices create noise in the evolutional dynamics, which implies that no state is perfectly absorbing. However, there two regions of the state space-one equitable, the other fractious-that every persistent: once the process enters such a region, it tends to stay there for a long period time. They showed that the emergence of the equity norm by self-interested agents is hard in the sense that it takes exponential time to achieve it from some initial states.

Table 1 The payoff matrix of the Nash demand game

\begin{tabular}{c|lr|rr|rr}
\hline Agent B & $\begin{array}{c}S_{1} \\
\text { (High) }\end{array}$ & $\begin{array}{c}S_{2} \\
\text { (Medium) }\end{array}$ & \multicolumn{2}{|c}{$\begin{array}{c}S_{3} \\
\text { (Low) }\end{array}$} \\
\hline \hline $\begin{array}{c}S_{1} \\
\text { (High) }\end{array}$ & 0 & 0 & & 0 & & 3 \\
\hline $\begin{array}{c}S_{2} \\
\text { (Medium) }\end{array}$ & 0 & & 5 & & 5 & \\
\hline $\begin{array}{c}S_{3} \\
\text { (Low) }\end{array}$ & 3 & 7 & & 5 & & 3 \\
\hline
\end{tabular}

\subsection{Dispersion Game: Generalized-Rock-Scissors Paper Game}

The hand game "Rock-Scissors-Paper (RSP)" is also known as “Janken” in Japan, has been around the world for a long time. It most often used to solve small conflicting matters between peoples but it can also be played to decide larger matters, as part of tournament, our simply as a diversion. The basics of the 
game consist of each player shaking a fist a number of times and then extending the same hand in a fist ("rock"), out flat ("paper"), or with the index and middle fingers extended ("scissors").

The RSP game is also important for the study in many ecosystems. Kerr et al set out to investigate the mechanisms that maintain biodiversity in ecosystems [10]. Studies of three bacterial strains engaged in an interaction that mimics the game, rock-scissors-paper, show the importance of localized interactions in maintaining biodiversity. Kerr and colleagues are not the first to show that localized interactions of the rock-scissors-paper type can turn a one winneroutcome into a dynamic coexistence of all three types, endlessly chasing each other across the board [21].

We consider a population of agents located a lattice network repeatedly play the generalized RSP games with the payoff matrix in Table 2. The generalized RSP game in Table 2 has the unique Nash equilibrium, and each strategy, rock, scissors and paper should be selected with the same probability $1 / 3$. The expected payoff of each agent with this mixed Nash equilibrium is $(\lambda+1) / 3$. Except the case the parameter $\lambda$ is greater than or equal to 2, the payoff at equilibrium is asymmetric and the problem of the fairness may arise.

Table 2 The payoff matrix of the generalized rock-scissors-paper game $(\lambda \geq 2)$

\begin{tabular}{|c|c|c|c|}
\hline Own Strategy & $\begin{array}{c}S_{1} \\
\text { (Rock) }\end{array}$ & $\begin{array}{c}S_{2} \\
\text { (Scissors) }\end{array}$ & $\begin{array}{c}S_{3} \\
\text { (Paper) }\end{array}$ \\
\hline $\begin{array}{c}S_{1} \\
\text { (Rock) }\end{array}$ & 1 & $\lambda$ & 0 \\
\hline $\begin{array}{c}S_{2} \\
\text { (Scissors) }\end{array}$ & 0 & 1 & $\lambda$ \\
\hline $\begin{array}{c}S_{3} \\
\text { (Paper) }\end{array}$ & $\lambda$ & 0 & 1 \\
\hline
\end{tabular}

\section{Strategy Choice Based on Learnable Behavioral Rule}

In orthodox rational choice theory, agents are modeled as cognitively sophisticated and entirely self-interested decision makers who evaluate every future consequence of possible actions and select the action alternative that maximizes their self-interest. Discrete choice analysis grounded in the theory of 
Akira Namatame

utility maximization has proven quite successful in terms of its usefulness. However, this approach is being challenged by a line of research originating in cognitive psychology that is causing economists to re-examine the standard model of choice behavior [13]. In the words of the psychologist Kahneman, economists have preferences; psychologists have attitudes [20]. However, Experimental evidence supports the view the behavioral rules are the proximate drivers of most human behavior. The rule-governed action can be pictured as a quasi-legal process of constructing a satisfying interpretation of the choice situation. The behavioral rules we do use are essentially defensive ones, protecting us from mistakes that perceptual illusions may induce. However, the question remains as to whether behavioral rules themselves develop in patterns that are broadly consistent with the rational model postulates. This is a vital scientific concern. If there are preferences behind the formation of behavioral rules, then how they are correlated with these underlying preferences.

We seldom do new things. Most behaviors are repeated, but many researchers do not pay much attention to this aspect. Few would dispute the claim that most behaviors are repetitive, yet in spite of a large literature on learning, the habit concept has received only minor attention. The sort of coordination problems we have in mind are those that we commonly solve without thought or discussionusually so smoothly and effortlessly that we don't even notice that there is a coordination problem to be solved. To operate successfully, a society needs to single out one of these on which to coordinate.

Verplanken and Aarts define habits as learned sequences of acts that have become automatic responses to specific cues, and are functional in obtaining certain goals or end-states [20]. Obviously, many behaviors may fall under this definition, varying from being very simple to being complex. Habits are learned sequences of acts. Habits are also automatic responses to specific cues. Habitual acts are instigated as immediate responses to specific cues. Importantly, these responses occur without purposeful thinking or reflection and often without any sense of awareness. Most habits are created and maintained under the influence of reinforcement learning. In other words, behavioral rule that has positive consequences is more likely to be repeated, whereas negative consequences make repetition less likely. Repeated behaviors may turn into habits, which are automatic responses to specific cues and are functional in obtaining certain goals. We may want new and desired behaviors to become habits, which makes them stable and difficult to change. Habituation may thus become a behavioral rule.

Social norms and habit influence in turn individuals' purposive behaviors based on their current preferences. This bi-directional causal relationship is at the essence of the study of cognitive science. Understanding the nature of the relationship between two different levels at which actual choice is also a grand challenge. Explanation of this relationship calls for examining the types of interactions that link individuals in social contexts. 
The Wisdom of Networked Evolving Agents

In this paper we propose a hybrid choice model based on both rule based choice and preference choice as shown in Figure 1. Agents adhere to behavior rules via local adaptation of behavior. The adaptation of behavior rules consists of an internalization of social norms, or more precisely a synchronization of the individual behavioral to those of the other neighbors. Each agent applies the hybrid choice model based on both agentspecific assessments of the situations (rational choice model) and rule based choice based on social norms. Social norms have been treated here as constraints on agent-specific rational choices. Each agent is modeled to evolve her behavioral rule. This hybrid choice model at individual levels is the core of socially intelligent behavior.

We stresses that the performance of the socio-economic system consisting of self-fish agents depends on how they are properly coupled. A strategy choice based on the behavioral rule for repeated play of the game uses the recent history of play to choose one of the three strategies for the next play. Here, we assume that each agent can remember the last outcome. Each behavioral rule is represented as a binary string so that the genetic operators can be applied. We represent a behavioral rule by a 3-bits string using $\mathrm{S} 1=0, \mathrm{~S} 2=1$ and $\mathrm{S} 3=2$. In order to accomplish this we use a bit string. Since no memory exists at the start of the game, extra one bit is needed to specify a hypothetical history at the beginning.

Each position $p_{j}, j=1, . ., 12$, in Figure 2 represents as follows. The first position $p_{1}$ encodes the initial strategy that the agent takes at each generation. A position $p_{j}, j=2,3$, encodes the history of mutual hands (rock, scissor, or paper) that agent and her opponent took at the previous round. A position $p_{j}, j=4, ., 14$, encodes the action that the agent takes corresponding to the values at the positions $p_{j}, j=2,3$.

There are nine possible outcomes for each round. We can fully describe a behavioral rule by recording what the strategy will do in each of the nine different outcomes that arise in the last play of the game. A rule must specify depending each outcome, what strategy the agent should choose at the net round. Since there re three strategies, the number of possible behavioral rules is $3^{9}$. The hope is that agents would find a better behavioral rule out of the overwhelming possible rules after a reasonable number of plays.

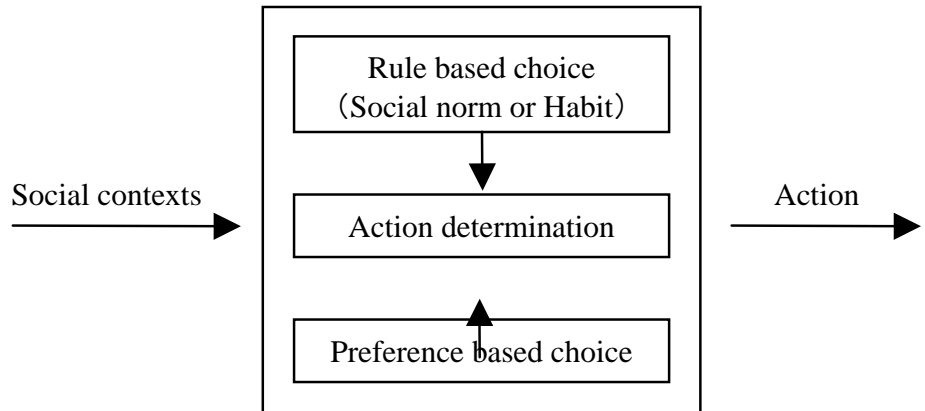

Figure 1 A Hybrid Choice Model 
Akira Namatame

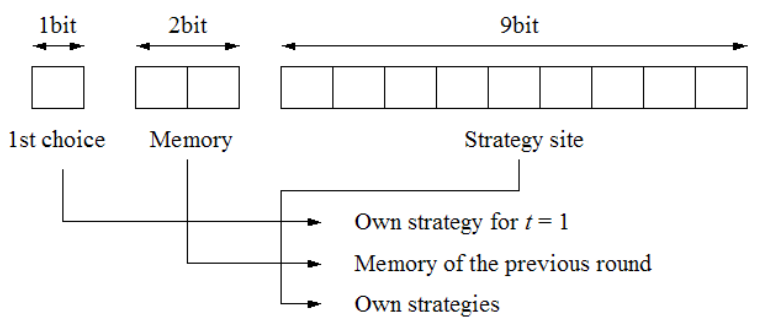

Figure 2 Memory Agent's of a behavioral rule

\section{Individual Learning vs. Social Learning}

To achieve desirable outcomes, a primary question is how each individual should learn in the context of many learners [22]. There are two competing approaches for describing the learning model of the population: the microscopic model based on individual learning and the macroscopic model based on social learning.

In the category of individual learning, agents are modeled to have some repertories of behavioral rules, and they update those rules using the existing rules within. Natural selection operates on the local probability distribution of behavioral rules within the repertoire of each individual agent. In an individual learning model, we could say that each agent checks if another randomly chosen agent in the population gets a higher payoff, and, if so, switches to that behavior with a probability proportional to the payoff difference.

There is no imitation or exchange their experiences among agents in individual learning. On the other hand, social learning becomes valuable in a social context, since it can help to surface new ideas and generate social consensus on issues that no single individual can effectively make right decision about alone. Social learning can also be extended beyond the boundaries of a single agent. Social learning is one that has an internal process for cultivating individual learning and connecting it to others. So when faced with change, a collective has the requisite energy and flexibility to move in the direction it desires.

In an orthodox social learning model, agents play based on the prescribed behavioral rules. The summed payoff of each game provides the agent's fitness. After every individual has played the game with her neighbors, each rule of the agents is updated according to the general evolutionary rules, and the behavioral rule is crossover with the most successful behavioral rule of her neighbors. Their success depends in large part on how well they learn from their neighbors. If an agent gains more payoff than her neighbor, there is a chance her behavioral rule will be imitated by others. 
The principle of social learning itself can be thought of as the consequence of any one of three different mechanisms. It could be that the more effective individuals are more likely to survive and reproduce. A second interpretation is that agents learn by trial and error, keeping effective rules and altering ones that turn out poorly. A third interpretation is that agents observe each other, and those with poor performance tend to imitate the rules of those they see doing better.

The most unrealistic aspect of the rule learning is the large number of strategies each agent considers. Even if the set of rules is limited to very simple ones, each agent remembers to many strategies. A realistic model should account for the fact that agents consider a much smaller number of rules from which they learn and make decisions; and that the rules agents consider are often preconditioned by factors such as imitation that have evolved over the generations.

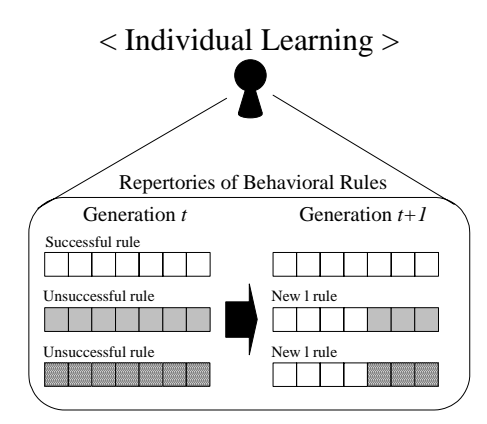

(a)

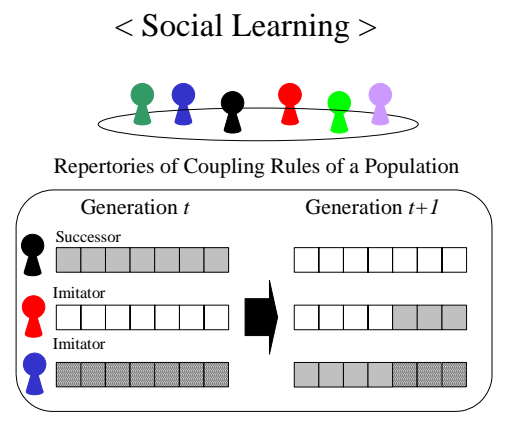

(b)

Figure 3 Individual learning vs. social learning of a behavioral rule

\section{Social Interactions of Networked Agents}

A crucial ingredient in social interaction models is the network structure in which individuals interact. The interaction structure specifies who affects whom, and this network structure may vary from one individual to another. The agents involved would learn two things: with whom to interact and how to behave. That is to say that learning dynamics operates both on network structure and strategy. The interaction structure specifies who affects whom.

In order to describe the ways of interaction, the random matching model is frequently used. In the random matching model, in which each agent is assumed to interact with a randomly chosen agent from the population. There are also a variety of interaction models, depending on how agents meet, and what information is revealed before interaction. 
Akira Namatame

There are many situations in which a spatial environment becomes a more realistic representation, since interactions in real life rarely happen on such a macro-scale as assumed in the global interaction model. Spatial interaction is generally modeled through the use of the two dimensional (2D) grid in Figure 5 with each agent inhabiting each cell of the lattice on the grid. Interaction between agents is restricted to nearest neighboring agents. Each agent chooses an optimal strategy based on local information about what her neighbors will choose. However, the consequences of their choices may take some time to have an effect on agents with whom they are not directly linked.

At another end of the spectrum we have models where individuals interact with both fixed their neighbors and randomly chosen agents from the population. Watts and Storogatz introduced a small-world network architecture that transforms from a nearest neighbor coupled system to a random coupled network by rewiring the links between the nodes [21]. For instance, consider a two-lattice model in which each node is coupled with its nearest neighbors, as shown in Figure 5. If one rewires the links between the nodes with a small probability, then the local structure of the network remains nearly intact.

If we fix the interaction structure, we get models of the evolution of strategies in games played on a fixed network structure. An interaction structure need not be deterministic. In general, it can be thought of as a specification of the probabilities of interaction with other individuals. By far the most frequently studied interaction structure is one in which the group of individuals is large and individuals interact at random. That is to say that each individual has equal probability of interacting with every other individual in the population.

Some researchers also concern the impact of different network structures on equilibrium selection in the context of coordination games. If agents can choose their partners to interact, then they will form networks that lead to play of the efficient Nash equilibrium in the underlying coordination game. Ellison analyzed the role of local interactions for the spread of particular strategies in coordination games, showing, how play converges to the risk-dominant equilibrium if agents are located on a circle and interact with their two nearest neighbors [7]. Similarly, Blume and Kosfel proved the convergence to the risk-dominant equilibrium in a population of agents located on a two-dimensional lattice [4][11]. Kuperman and Abramson studied an evolutionary version of the prisoner's dilemma game, played by agents placed in a small-world network [12]. Agents are able to change their strategy, imitating that of the most successful neighbor. They found that collective behaviors corresponding to the small-world network enhances defection where cooperation is the norm in the fixed regular network.

Ultimate interest resides in the general case where structure and strategy coevolve. These may be modified by the same or different kinds of learning. They may proceed at the same rate or different rates. The case where structure dynamics is slow and strategy dynamics is fast may approximate more familiar 
models where strategies evolve on a fixed interaction structure. Whether coevolution of structure and strategy supports or reverses the conventional wisdom about equilibrium selection in this game, depends on the nature and relative rates of the two learning processes.

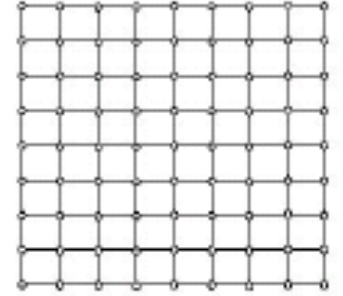

(a)

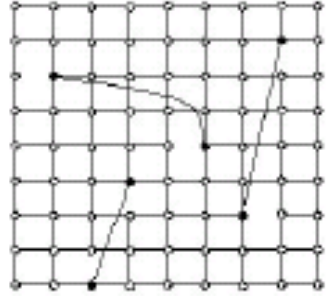

(b)

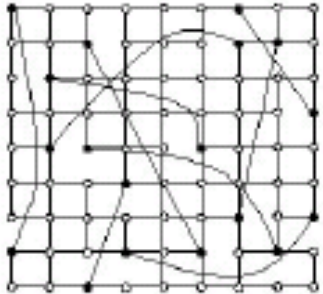

(c)

Figure 4 Social interactions of networked agents. (a) $p=0$, a regular lattice. (b) $p=0.1$, some of the links have been re-wired resulting in a small-world network. (c) $p=0.2$, additional re-wiring has occurred. As $\mathrm{p}$ approaches 1 , a transition to a random network will occur.

\section{Simulation Results}

\subsection{Nash Demand Game}

Figure 5 shows (i) the average payoff per agent and (ii) the ratio of each strategy over generation when each agent repeatedly plays the Nash demand game with the payoff matrix in Table 1 . After the 12 generation, the average payoff is increased to 5 , and every agent chooses $S_{2}$ (medium). Many spheres of social interactions are governed by social norms such equity. In this case, the efficient and equity norm is emerged over the networked agents.

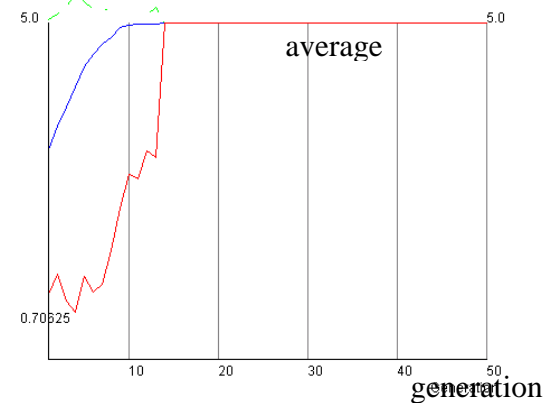

(i) The average payoff per agent

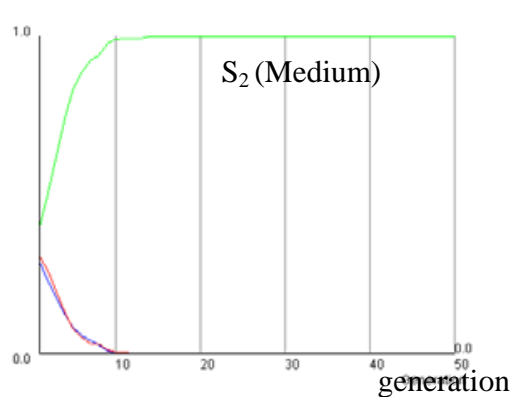

(ii) the ratio of each strategy

Figure 5 Simulation results of Nash demand games 
Akira Namatame

\subsection{Generalized RSP Game}

We simulated several cases by changing the parameter value of $\lambda$. We also consider the effect of implementation error. That is, there is small probability of choosing the wrong strategy specified the rule. Significant differences will be observed when agents have small chances of making mistakes.

(Case 1) $\lambda=2$ : Figure 6 shows (i) the average payoff per agent and (ii) the ratio of each strategy over generation when we set $\lambda=2$ in Table 2 . All agents receive the same average payoff if $\lambda=2$ by choosing $\mathrm{S}_{2}$ (scissors).

(Case 2) $\lambda=10$. We now investigate the strategic situation by increasing the payoff of winning the game by setting $\lambda=10$ in Table 3 . Figure 7 shows the simulation results without any implementation error. Figure 7(a) shows the payoff per agent at each generation, and there exit lucky agents who gain the highest payoff, which is close to 10 , by always winning the game and also unlucky agents who gain almost nothing by always losing the game. The average payoff per agent is approximately 3.9, which is higher than the expected payoff at Nash equilibrium, which is approximately 3.7. In the beginning, 400 different coupling rules were aggregated into three types, as shown in Table 5.

We also investigate what behavioral rules agents learned by repeatedly playing RSP games. The behavioral rules learned by 400 agents are shown in Table 3. The number of the right column shows the number of the agents who have learned the same rule.

The game between two agents who play with the behavioral rules can be described as a stationary stochastic process. The state transition of the outcomes when both agents choose their strategies according to the same coupling rule of type 1 is illustrated in Figure 8 as the state transition diagram.

(Case 1) Agents who have the same behavioral rule

The strategy choices between two agents with the same coupling rule type $i$, $i=1,2, . ., 8$, are shown in Figure 6(a). In this figure, there is one absorbing state at " 00 " and one limiting cycle. The state diagram contains two paths, one for moving towards to the absorbing state and one for the limiting cycle, and there is no path between the two cycles. As shown in Table 3, agents also learn to initiate the play by choosing paper (2) and strategy choices eventually converge to 00 . This means that if an agent plays with other agents of the same rule, they converge to the state of a tie, and receive the lower payoff of 1 .

(Case 2) Agents who have the different behavioral rules: We now investigate the state diagrams of plays by two agents who have different coupling rules in Table 3. The state diagram is shown in Figure 6(b). If the system were to start from the set of the states, it would evolve to an attractor. These are known as the basin of attraction. In this case, the point attractor for the state of the systems is replaced by a circle, and in the limit, the system moves endlessly around this 
The Wisdom of Networked Evolving Agents

circle. Starting from any state, it eventually converges to an efficient cycle such that agents win three times and lose three times.

With some mistakes, some interesting properties emerge. If an agent $A$ chooses rock (0) and her opponent $B$ chooses "scissors (1)" (in this case she wins and her opponent loses), then in the next round agent $A$ chooses "scissors (1)" and agent $B$ chooses "paper (2)". In the following round agent $A$ chooses "paper (2)" and agent $B$ chooses "rock (0)". Therefore, agent $A$ wins three times and agent $B$ loses three times. However, after these games, the two agents completely reverse roles, and the winning agent thus far, agent $A$, chooses "scissors (1)" and the losing agent thus far, agent $B$, chooses "rock (0)". After these three one-sided games, they trade places. The winner thus far chooses "scissors (1)" and loser thus far chooses "rock (0)". The winner then becomes the loser, and vice versa. In total, her opponent wins three times. Both agents are eventually absorbed into the limit cycle of the three-wins and three-losses. Thus far, this agent wins three times and her opponent loses three times. Therefore, the two agents switch roles as winner and loser. Since both agents win three times and lose three times, on the average, they gain the payoff at Pareto-efficiency.

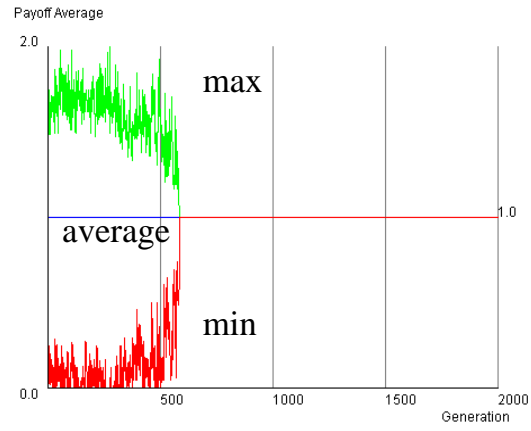

(i) The average payoff per agent

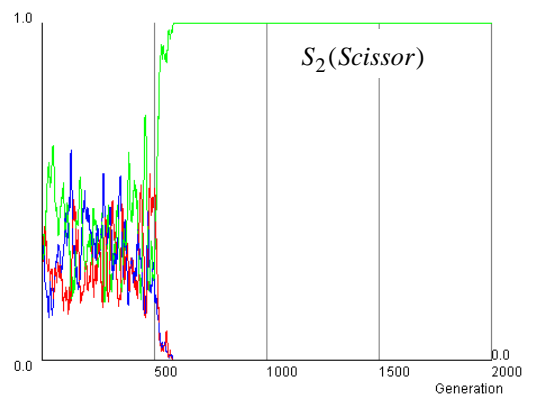

(ii) the ratio of each strategy

Figure 6 Simulation results with $\lambda=2$

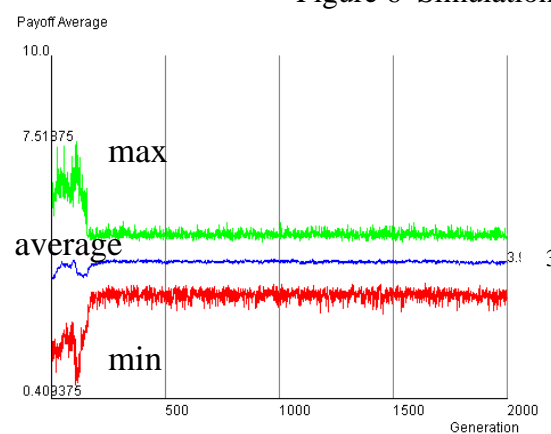

(i) The average payoff per agent

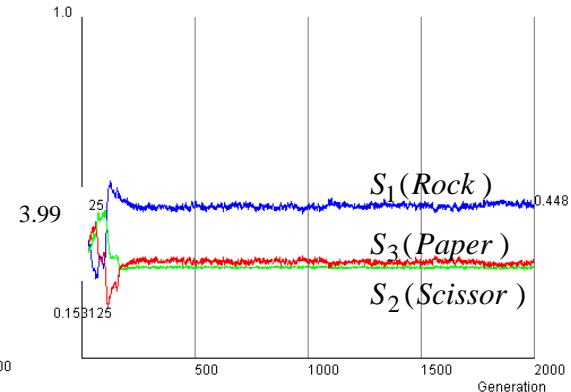

(ii) the ratio of each strateg

Figure 7 Simulation results with $\lambda=10$ implementation error: $10 \%$ 
Akira Namatame

Table 3 Learnt behavioral rules by 400 agents

\begin{tabular}{|c|c|c|c|c|c|c|c|c|c|c|c|}
\hline \multirow{3}{*}{ Rule type } & $\begin{array}{c}\text { Initial } \\
\text { Strategy }\end{array}$ & \multicolumn{9}{|c|}{ Strategy site } & \multirow{2}{*}{$\begin{array}{c}\text { Number of } \\
\text { agents }\end{array}$} \\
\cline { 2 - 13 } & 1 & 2 & 3 & 4 & 5 & 6 & 7 & 8 & 9 & 10 & \\
\hline \hline 1 & 2 & 0 & 1 & 0 & 2 & 0 & 2 & 1 & 0 & 0 & 149 \\
\hline 2 & 2 & 2 & 1 & 0 & 2 & 0 & 2 & 1 & 0 & 0 & 102 \\
\hline 3 & 2 & 0 & 1 & 0 & 2 & 2 & 2 & 1 & 0 & 0 & 58 \\
\hline 4 & 2 & 2 & 1 & 0 & 2 & 2 & 2 & 1 & 0 & 0 & 41 \\
\hline 5 & 2 & 2 & 1 & 0 & 2 & 0 & 2 & 1 & 0 & 2 & 20 \\
\hline 6 & 2 & 0 & 1 & 0 & 2 & 0 & 2 & 1 & 0 & 2 & 15 \\
\hline 7 & 2 & 0 & 1 & 0 & 2 & 2 & 2 & 1 & 0 & 2 & 9 \\
\hline 8 & 2 & 2 & 1 & 0 & 2 & 2 & 2 & 1 & 0 & 2 & 6 \\
\hline
\end{tabular}

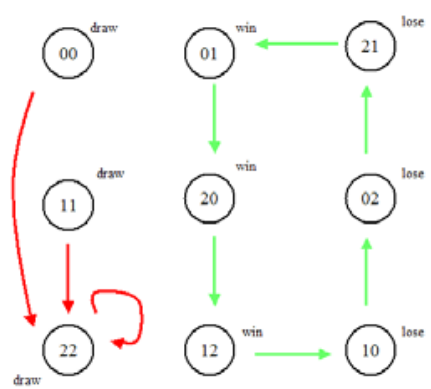

(a)

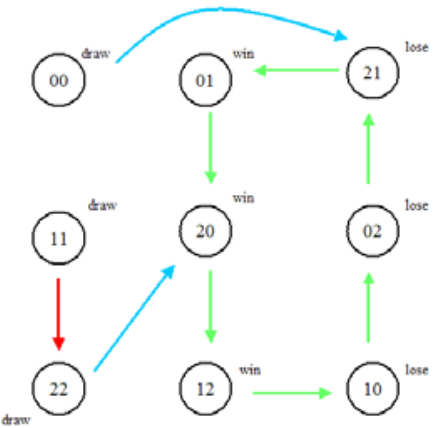

(b)

Figure 8 The state diagram of play between two agents with the same rule Agents who have the behavioral rules (a) Agents who have the same behavioral rules, (b) Agents who have the different behavioral rules

\section{Social Norms Emerged over Networked Agents}

Many laboratory experiments and field observations indicate that humans are social animals who take a strong interest in the effects of their actions on others and whose behavior is not always explained by simple models of selfish behavior. Reciprocity and the presence of norms can support a great deal of social intelligent behavior. 
The simulation results in the previous section implicate that we have a tools for examination how social norms evolve in a society that begins in an amorphous state where there is no established common behavioral rules and individuals only rely on hearsay to determine what to do. We discussed how various social norms emerge in a society of interacting agents. Especially, we investigate the conditions under which the norm will emerge and dominate in various social setting, and discuss the importance of collective norm construction for evolving and sustaining a desirable society of efficiency and equity. After collective construction of social norms, there is no need to assume a rational calculation to identify the effective behavioral rule. Instead, the analysis of what is chosen at any specific time is based upon an implementation of the idea that effective behavioral rules are more likely to be retained than ineffective ones.

Epstein extended the literature on the evolution of norms with an agent-based model [8]. In his model, agents learn how to behave (what norm to adopt), but they also learn how much to think about how to behave. The point of his model is that many social norms or conventions have two features of interest. First, they are self-enforcing behavioral regularities. But second, once entrenched, we conform without thinking about it. Indeed, this is one reason why social norms are useful; they obviate the need for a lot of individual computing.

The asymmetry in payoffs from interaction induces agents to learn the behavioral rule that breaks the asymmetry. We consider the generalized RSP game in which favourable payoffs are possible only if one agent acts one way while the other acts the opposite way. To coordinate successfully, the agents have to alternate or take turns, out of phase with each other. If this type of social interaction is repeated, the agents benefit by coordinated alternation by taking turns in choosing one of the three strategies and there is evidence to show that this type of turn-taking occurs quite commonly in nature. Give-and-take is a strategy that is intuitive and simple, but even so it is beyond the scope of most traditional learning models.

Hanaki used adaptive models to understand the dynamics that lead to efficient and fair outcomes in a repeated battle of the sexes game [9]. He develops a model that not only uses reinforcement learning but also the evolutionary learning that operates through evolutionary selection. He found that the efficient and fair outcome emerges relatively quickly through turn taking. However, his model requires a long run pre-experimental phase before it is ready to take turn. Turn taking in the battle of the sexes game is just one of many game theoretic phenomena, and it raises an important general point for further studies.

Browning and Colman also investigated how coordinated, alternating cooperation can evolve without any communication between agents who play battle of the sexes game [5]. They study the nature, properties and phenomena of coordinated alternating cooperation in a range of dispersion games with asymmetric equilibria. By alternating coordination the agents benefit from it, 
Akira Namatame

however, how agents evolves alternating coordination without communication is not fully explained.

\section{Conclusion}

Networks of evolving agent are likely to foster social interactions where individual self-interest is consistent with behavior that maximizes the social. Social interaction in such network structure is best modeled as a repeated game. In repeated games, where an agent's actions can be observed and remembered by other agents, almost any pattern of individual behavior, including behavior that maximizes group payoff, can be sustained by social norms that include obligations to punish norm violations by others. Where many equilibria are possible, co-evolution is likely to play a major role in determining Paretoefficient equilibrium will obtain.

We analyzed the emergent and aggregate behavior of self-interested agents in social spaces. Our problem is to explain how such socially intelligent behaviour could have evolved, given that natural selection operates at the individual level. The framework of collective evolution distinguishes from the concept of coevolution in three aspects. First, there is the coupling rule: a deterministic process that links past outcomes with future behavior. The second aspect, which is distinguished from individual learning, is that agents may wish to optimize the outcome of the joint actions. The third aspect is to describe how a coupling rule should be improved with the criterion of performance to evaluate how the rule is doing well.

The performance assessment at the individual levels gradually evolves, in order for the agent to act in accordance with the behaviors of her neighbors. Social norms are not merely the union of the local behavioral rules of all agents, but rather evolve interactively, as do the local behavioral rules of the agents. In an evolutionary approach, there is no need to assume a rational calculation to identify the best behavioral rule. Instead, the analysis of what is chosen at any specific time is based upon an implementation of the idea that effective behavioral rules are more likely to be retained than ineffective ones.

\section{References}

1. Adamatzky, A. (2005). Dynamics of Crowd-Minds, World Scientific,

2. Axtell,R and Epstein,M, and Young, P. (2001). The emergence of classes in a multiaget bargaing model in. Social Dynamics, , Durlauf, N. and Young, P (eds). Brookings Institution Press, pp.191-211.. 
3. Bergstrom, T. (2002) Evolution of Social Behavior: Individual and Group Selection, Journal of Economic Perspectives, Volume 16, pp. 67-88

4. Blume, E. (1993). The statistical mechanics of strategic interaction, Games and Economic Behavior, 5, pp. 387-424.

5. Browning, L.and Colman, M. (2004). Evolution of coordinated alternating reciprocity in repeated dyadic games. Journal of Theoretical Biology 229,pp 549-557

6. Dick, S, Krambeck, M, and Milinski, S (2003) Volunteering leads to rock-paperscissors dynamics in a public goods game, Nature. Vol. 425, pp390-393

7. Ellison, G. (1993). Learning local interaction, and coordination, Econometrica, 61, pp.1047-1071.

8. Epstein, J. M. and Axtell, R. (1996). Learning to be thoughless: Social norms and individual computation, Working paper no.6, Brookings Institution, 1999.

9. Hanaki, N, Sethi, R, Erev. I and Peterhansl Learning Strategies. Journal of Economic Behavior and Organization, Vol. 56, pp.523-542, 2005.

10. Kerr, B, Riley, M, Feldman, Brendan, M. and Bohannan, M. (2002). Local dispersal promotes biodiversity in a real-life game of rock-paper-scissors, Nature, Vol. 418, pp 171-174.

11. Kosfeld, M. (2002). Stochastic strategy adjustment in coordination games, Economic Theory, 20, pp. 321-339.

12. Kuperman and Abramson (2001), Social games in a social network, Phys. Rev. E 63.

13. Manski, C. F. and McFadden, D. L. (Eds) (1981). Structural Analysis of Discrete Data and Econometric Applications, The MIT Press.

14. McMahon,C. (2003). Collective Rationality and Collective Reasoning, Cambridge University Press.

15. Namatame, A. (2006) Adaptation and Evolution in Collective Systems, World Scientific

16. Nowak, M. A. and Sigmund, K. (2004). Evolutionary dynamics of biological games, Science, 303, pp. 793-799.

17. Roughgarden, T. (2005). Selfish Routing and the Price of Anarchy, The MIT Press.

18. Surowiecki, J. (2004). The Wisdom of Crowds, Random House.

19. Skyrms, B and Pemantleá, R. (2000) A dynamic model of social network formation,

PNAS, vol. 97, pp.9340-9346

20. Verplanken B, Aarts H (1999). Habit, attitude, and planned behavior: is habit an empty construct or an interesting case of goal-directed automaticity? European Review of Social Psychology, Vol.10, pp.101-134.

21. Watts, D. and Strogatz, H. (1998). Collective dynamics of small-world networks, Nature, 393, pp. 440-442.

22. Young, H. P. (2005). Strategic Learning and Its Limits, Oxford Univ. Press. 